The study used an observational approach. Researchers filmed 55 mothers playing with their infants, and coded the mother-infant interactions for 'parent sensitivity' and 'parent maintaining'. They then assessed the infants' cognition and language ability annually. With the data in hand, they tested whether parenting style was linked with developmental outcomes, while controlling for vision level. The analytical approach chosen "allowed us to take into account that each infant has an individual rate of development, which is the case in a very heterogeneous sample of infants with vision disorders", explains Dale.

The researchers found that higher maternal sensitivity was linked with increased verbal comprehension from infancy to age 3 years. "This is of interest because verbal comprehension is more at risk in infants and young children with visual impairment, as the child cannot see the 'language referent' visually and may respond to language without having semantic understanding", says Dale. "It would suggest that early parent-infant interactions provide a socio-emotional framework to help the infant with visual impairment learn more about the world around them, as language is key for helping these young children access the world".

Going forward, Dale and colleagues propose that supporting parents to interact sensitively with visually impaired infants might help their children's longer term language development and understanding of the world around them. This insight will likely help to design more effective early interventions for parents of infants with visual impairments. "Our larger OPTIMUM study showed that a structured developmental framework (the Developmental Journal for babies and young children with visual impairment) fostered greater sensorimotor understanding and expressive language development whereas this study showed that parenting style fostered greater verbal comprehension development", concludes Dale. "Now, we are starting to understand the different needs that may benefit from early intervention and to support the complex development of these infants".

\section{Referring to:}

Sakkalou, E., O'Reilly, M.A., Sakki, H., Springall, C., de Hann, M., Salt, A.T. \& Dale, N.J. (2020), Motherinfant interactions with infants with congenital visual impairment and associations with longitudinal outcomes in cognition and language. J. Child Psychol. Psychiatr. doi: 10.1111/jcpp.13308.

See also:

https://www.optimumstudy.org/

Glossary:

Parent maintaining: holding the child's interest and focus of attention

Parent sensitivity: empathic and accepting responses to the infant's cues about their mental states

\section{"We set out to undertake an ambitious national, longitudinal study (the OPTIMUM project), to ensure sufficient recruitment of 8-12 month infants with rare eye disorders and chronic visual impairment who we could follow through a 2-year period (with three time points)."}

